\title{
A method for stabilization of drone flight controlled by autopilot with time delay
}

\author{
Shiran Avasker ${ }^{1} \cdot$ Alexander Domoshnitsky $^{1} \cdot$ Max Kogan $^{1} \cdot$ Oleg Kupervasser $^{1,2} @ \cdot$ Hennadii Kutomanov $^{1}$. \\ Yonatan Rofsov ${ }^{1} \cdot$ Irina Volinsky ${ }^{1} \cdot$ Roman Yavich $^{1}$
}

Received: 20 August 2019 / Accepted: 4 January 2020 / Published online: 17 January 2020

(c) Springer Nature Switzerland AG 2020

\begin{abstract}
In this paper, the methods of the stability theory of differential equations with time delays are used in the study of an actual engineering problem of a drone (UAV) autonomous flight. We describe correct operation of autopilot for supply stability of desirable drone flight. There exists a noticeable delay in getting information about position and orientation of a drone to autopilot in the presence of vision-based navigation (visual navigation). In spite of this fact, we demonstrate that it is possible to provide stable flight at a constant height in a vertical plane. We describe how to form relevant controlling signal for autopilot in the case of the navigation information delay and provide control parameters for particular case of flight.
\end{abstract}

Keywords Visual navigation · Drone · UAV · Autopilot · Vision-based navigation · Time delay · Stability · Differential equations

\section{Introduction}

The methods of the stability theory of differential equations with time delays are applied for solving actual engineering problem of a drone (UAV) autonomous stable flight.

The main achievements of this paper are the following:

1. An example of adaptation of the mathematical theory (which was during long time developed without any connection to physics or engineering) in solving actual engineering problem.

2. The approach, proposed here for solving stabilization problem of drone autonomous flight, is much better than previously used ones in approximate engineering solution.

3. The adaptation of this mathematical theory for drone fight stabilization is a difficult problem, since the mathematical theory cannot be applied directly and explicitly for the system describing the drone motion. Indeed, we need to apply some nontrivial mathematical transform to the physical differential equations to make such use possible.

4. Even after getting from the mathematical theory constrains for controlled parameters defined by autopilot (which are necessary for stabilization drone flight), it is also nontrivial problem to find a solution for these parameters.

This paper is engineering application of mathematical stability theory for differential equations with delays described in [1-5]. Although there is a corresponding resistance of engineers to the use of theoretical results on stability of differential equations with delays instead of engineering approximate solutions, the theory of these equations develops intensively. Every year, hundreds of

Oleg Kupervasser, olegkup@yahoo.com | 1 Department of Mathematics, Ariel University, Ariel, Israel. ${ }^{2}$ TRANSIST VIDEO LLC, Skolkovo, Moscow, Russia. 
papers on stability analysis of delay equations are published. Let us start with two examples:

Example 1 These two very similar equations arise directly from the Newton second low: $y^{\prime \prime}(t)=0$ and $x^{\prime \prime}(t)=\varepsilon$. Assume that $\varepsilon$ is very small and consider the same initial conditions at the point 0 : $y(0)=0, y^{\prime}(0)=0$ and $x(0)=0, x^{\prime}(0)=0$. Their solutions are $y(t) \equiv 0$ and $x(t)=\frac{\varepsilon}{2} t^{2}$ respectively. It is clear that $\lim _{t \rightarrow \infty} x(t)-y(t)=\infty$, and there is no stability with respect to right-hand side. Conclusion: we need a feedback control to stabilize the equation $x^{\prime \prime}(t)=f(t)$ with respect to a right-hand side. In this way, we come to the stability analysis of the delay equation $x^{\prime \prime}(t)=-\sum_{i=1}^{m} a_{i} x\left(t-\tau_{i}(t)\right)$. $\tau_{i}(t)>0$ appeared as a result of information, operation or transport delays existing in all real technological processes.

Example 2 There exists a delusion that instead of stability analysis of the delay equation, one can use elements of the modern technology, for example, GPS gives us the values of $x\left(t_{1}\right), x\left(t_{2}\right), x\left(t_{3}\right), \ldots$ with very small time intervals $\Delta t_{i}=t_{i+1}-t_{i}$. It can be demonstrated, for example, on the delay equation $x^{\prime \prime}(t)=-a x([t])$, where $[t]$ is the integer part of $t$ and $a>0$. At the moment $t$, we know almost exactly $x([t])$, but this does not help in stabilization. Actually, it is known from the paper [6] that all solutions of the equation $x^{\prime \prime}(t)=-a x(t-\tau(t))$ are bounded if and only if $\int_{0}^{\infty} \tau(t) \mathrm{d} t<\infty$. For our equation $x^{\prime \prime}(t)=-a x([t])$, we have $\tau(t)=t-[t]$ and $\int_{0}^{\infty} \tau(t) \mathrm{d} t=\infty$. This means that there exist unbounded solutions of the equation $x^{\prime \prime}(t)=-a x([t])$, and this equation is unstable. The direct use of GPS without theoretical basis could not achieve stabilization even the signals from GPS come with very small time intervals. Results on exponential stability, i.e., all solutions of the homogeneous equation $x^{\prime \prime}(t)=-\sum_{i=1}^{m} a_{i} x\left(t-\tau_{i}(t)\right)$ tend to zero like exp $(-\alpha t)$ with positive $\alpha$, were obtained for the case $m \geq 2$ under corresponding conditions on the coefficients and delays in the form of inequalities in the paper [4].

Stability analysis presents one of the necessary parts in the almost all papers on robotics. Their authors avoid considering the delay in their models although they accept fact of arising transport, information, or executive delay in robotics models. The technique of Lyapunov's functions which has the long history (starting with works of N.Krasovskii in 1950s) is usually used but is not convenient in many cases for stabilization by delay feedback control.

The current basic engineering method for analyzing a delayed system is replacing the system with delays to the system without delay and using the classical theory of stability (characteristic equations in linear case, and method of Lyapunov's functions-in nonlinear). It is usually achieved in the frame of the following two ideas or their combinations [7-9]:

(A) to extrapolate a motion forward during the delay time,

(B) to take into account the estimate error of a current state and to use all possible values of the process for its future analysis.

The use of method (B) results in an obvious decrease in the accuracy of control and its effectiveness.

The use of method $(A)$ is possible when the system is sufficiently inert and does not have a strong control effect during the delay time. Even in this case, we need to use a complex algorithm. This results in an increase in the time and cost of creating a control system, the cost of computing power for extrapolation. The simplification of the model leads to a decrease in the accuracy and effectiveness of control. Also, when we change and upgrade the system, this big work needs to be carried out again.

If there is a control effect on the behavior of the system during the delay time, the method makes even more expensive and complex-it requires complex iterative schemes, the iterations do not always converge and require a long calculation time, which may insert an additional time delay. This can lead to a complete loss of controllability of the system. In order not to be unfounded from the mathematical point of view, we added an explanation of the shortcomings of approach $(A)$ and the justification for the necessity of using new results on stability theory.

It looks that the use of Azbelev's theory of stability of functional differential equations can open new perspectives in the control in robotics (see the book [10]). In the book [2], based on Azbelev's theory, there was developed the stability analysis and methods of estimates of solutions to systems of delay differential equations. The following can be noted as advantages of this approach:

1. It reduces the time and costs for control development.

2. It is easy to modernize the control of the system if the system has been changed.

3. Methods are universal for a wide class of systems.

4. Due to the high accuracy of mathematical methods, the system will have efficient and precise control.

5. There are no additional delays or control failures for complex cases where the controlled system is not inertial, and the control effect is significant during the delay time.

In this paper, we describe correct operation of autopilot for supply desirable drone flight (movement of a drone in a 
vertical plane at a constant height). For the finding drone, flight parameters were used vision-based navigation [11-20]. For realization vision-based navigation was developed the computer program "Video-navigation of UAV over relief" [11]. This program was tested in Zhejiang Province in east China near the capital Hangzhou using Google Earth data [17]. There always exists a noticeable delay in getting information about position and orientation of a drone to autopilot for vision-based navigation because of computer processing image's big data. In spite of this fact, we demonstrate that it is possible to provide stable flight at a constant height in a vertical plane. We want to describe how to form relevant controlling signal for autopilot in the case of the navigation information delay. We plan to use the autopilot described in the paper for controlling flight parameters found from vision-based navigation.

In this paper, description of motion equations and parameters of drone flight controlled by autopilot is based on the results of the book [21].

The structure of the paper is the following. The first section is Introduction. We described here the theme of the paper - state of the art with references in the field of stability theory methods and methods of description for drone flight controlled by autopilot with delay. The second section gives detailed preliminary results for mathematical stability theory methods which are used for drone flight controlled by autopilot in this paper. The model of drone flight is described. In the third section, we use the mathematical stability theory for finding parameters controlled by autopilot which are necessary for drone flight stability and finding upper boundary for the time delay. The theoretical results are compared with results of computer simulations. The fourth section is conclusion.

\section{Preliminary results of the investigation for drone flight stability}

\subsection{Mathematical preliminary results: stability of systems with time delays}

Throughout the paper, " $e$ " denotes the Euler number. $L_{\infty}$ is the space of essentially bounded measurable functions: $[0,+\infty) \rightarrow R$.

Consider the non-homogenous system of differential equations

$$
\begin{aligned}
& \left.x_{i}^{\prime}(t)+\sum_{j=1}^{n} \sum_{k=1}^{m} a_{i j}^{k}(t) x_{j}\left(t-\theta_{i j}^{k}(t)\right)=0, t \in 0,+\infty\right) \\
& x(\xi)=0, \xi<0, i=1, \ldots, n,
\end{aligned}
$$

where $A_{k}(t)=\left\{a_{i j}^{k}(t)\right\}_{i, j=1, \ldots, n}$ are $n \times n$ matrices with entries $a_{i j}^{k}(t) \in L_{\infty}, \theta_{i j}^{k}(t) \in L_{\infty}$ for $k=1, \ldots$, mand $i, j=1, \ldots, n$. The components $x_{i}:[0,+\infty) \rightarrow \mathbb{R}$ of the vector $x=\operatorname{col}\left\{x_{1}, \ldots, x_{n}\right\}$ are assumed to be absolutely continuous and their derivatives $x_{i}^{\prime} \in L_{\infty}$. A vector function $x$ is a solution of (2.1) if it satisfies system (2.1) for almost all $t \in[0,+\infty)$.

Let us denote

$$
\begin{aligned}
\theta_{i i}^{+}(t) & =\max _{m \geq k \geq 1} \theta_{i i}^{k}(t) \\
\theta_{i i}^{-}(t) & =\min _{m \geq k \geq 1} \theta_{i i}^{k}(t) \\
\Delta_{i} & =\operatorname{esssup}_{t \geq 0}\left\{\theta_{i i}^{+}(t)-\theta_{i i}^{-}(t)\right\} .
\end{aligned}
$$

It was shown in Theorem 3.2 in [1] that: If the following conditions are fulfilled:

1.1 There exist positive numbers $z_{1}, \ldots, z_{n}$ such that $\sum_{k=1}^{m} a_{i j}^{k}(t) z_{i}-\sum_{j=1, j \neq i}^{n} \sum_{k=1}^{m}\left|a_{i j}^{k}(t)\right| z_{j} \geq 1, t \in[0,+\infty), i=1, \ldots, n$

1.2 For every $i=1, \ldots, n$, at least one of the conditions (1.2a) or (1.2b) is fulfilled:

1.2a There exists $m_{i}$ such that $a_{i i}^{k}(t) \geq 0, a_{i i}^{j}(t) \leq 0$, $\theta_{i i}^{k}(t) \geq \theta_{i i}^{j}(t)$ for $k=1, \ldots, m_{i}, j=m_{i+1}, \ldots, m$, $\sum_{k=1}^{m_{i}} a_{i i}^{k}(t) \geq \frac{1}{e} \sum_{j=m_{i}+1}^{m}\left|a_{i i}^{j}(t)\right|$ for $t \in[0,+\infty)$ $\int_{t-\theta_{i i}^{+}(t)}^{t}\left\{\sum_{k=1}^{m_{i}} a_{i i}^{k}(s)-\frac{1}{e} \sum_{j=m_{i}+1}^{m}\left|a_{i i}^{j}(s)\right|\right\} \mathrm{d} s \leq \frac{1}{e} \quad$, $t \in[0,+\infty)$ and

1.2b There exists $m_{i}$ such that $a_{i i}^{k}(t) \geq 0, a_{i i}^{j}(t) \leq 0$, $\theta_{i i}^{k}(t) \leq \theta_{i i}^{j}(t)$ for $k=1, \ldots, m_{i}, j=m_{i+1}, \ldots, m$, $\sum_{k=1}^{m_{i}} a_{i i}^{k}(t) \geq \sum_{j=m_{i}+1}^{m}\left|a_{i i}^{j}(t)\right| \quad$ for $\quad t \in[0,+\infty)$ $\int_{t-\theta_{i i}^{+}(t)}^{t}\left\{\sum_{k=1}^{m_{i}} a_{i i}^{k}(s)-\sum_{j=m_{i}+1}^{m}\left|a_{i i}^{j}(s)\right|\right\} \mathrm{d} s \leq \frac{1}{e} \quad$, $t \in[0,+\infty)$ and $\int_{s}^{s+\Delta_{i}} \sum_{k=1}^{m_{i}} a_{i i}^{k}(\xi) \mathrm{d} \xi \leq \frac{1}{e} \quad \forall s \geq 0$, where $\Delta_{i}=\operatorname{esssup}_{t \geq 0}\left\{\theta_{i i}^{+}(t)-\theta_{i i}^{-}(t)\right\}$

Then system (2.1) is exponentially stable.

\subsection{Engineering preliminary results: drone's motion equations}

\subsubsection{Nonlinear equations}

Let us define the following variables and parameters used in equations of motion for a drone (see Fig. 1) [21]:

1. For forces and moments of forces:

$P$-Tractive force directed along longitudinal drone axis 


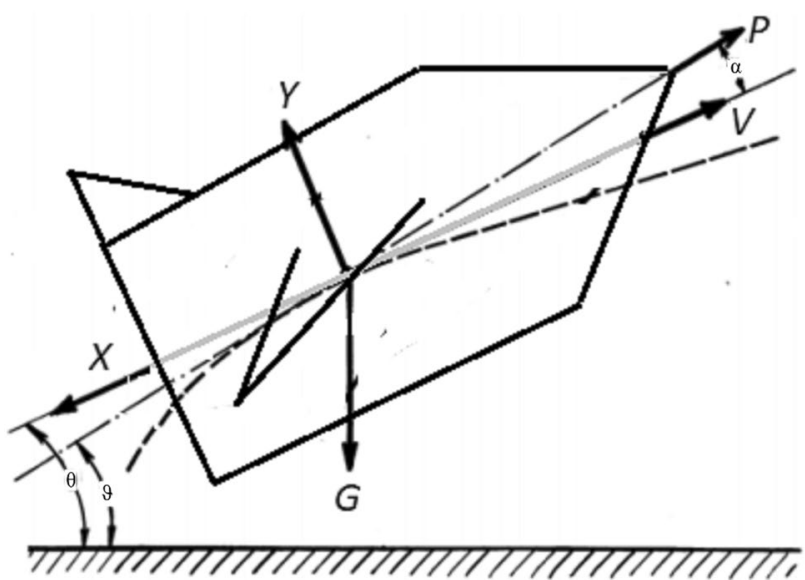

Fig. 1 Parameters of drone's longitudinal motion

$Y$-Carrying force orthogonal to flight velocity

$X$-Resistance force opposite to $V$

G-Gravitation force

$M_{z}$-Total moment of aerodynamical forces with respect of transversal axis

$f_{1}, f_{2}, f_{3}$-Random forces and random moments of forces
$m=G / g$-Drone mass

$J_{z}$-Inertial moment of drone with respect of axis $z p$-air density

4. Controlling signals:

$\delta_{p}$-Position of drone central control knob $\delta_{B}$-Deviation of drone control elevator

5. External environment parameters:

$U_{x}$ and $U_{y}$ - wind velocities along axes $x$ and $y$, correspondently

It is shown in [21] that forward movement and rotation are described by the system of equations:

$$
\begin{aligned}
m \frac{\mathrm{d} V}{\mathrm{~d} t} & =P\left(\delta_{p}(t), M(V, H)\right) \cos (\alpha)-X(\alpha, V, H)-G \sin (\theta)+f_{1}(t) \\
m V \frac{\mathrm{d} \theta}{\mathrm{d} t} & =P\left(\delta_{p}(t), M(V, H)\right) \sin (\alpha)-Y(\alpha, V, H)-G \cos (\theta)-f_{2}(t) \\
J_{z} \frac{\mathrm{d}^{2} \vartheta}{\mathrm{d} t^{2}} & =M_{z}\left(\alpha, M(V, H), \dot{\alpha}, \dot{\vartheta}, \delta_{B}(t)\right)+f_{3}(t) \\
\frac{\mathrm{d} H}{\mathrm{~d} t} & =V \sin (\theta)+U_{y}(t) \\
\frac{\mathrm{d} L}{\mathrm{~d} t} & =V \cos (\theta)+U_{x}(t) \\
\vartheta & =\theta+\alpha
\end{aligned}
$$

$$
\begin{aligned}
P & =P\left(\delta_{p}, V\right), X=c_{x}(\alpha, M) S \frac{\rho(H) V^{2}}{2}, Y=c_{y}(\alpha, M(V, H)) S \frac{\rho(H) V^{2}}{2}, \vartheta=\theta+\alpha, \\
M_{z} & =m_{z}\left(\alpha, M(V, H), \dot{\alpha}, \dot{\vartheta}, \delta_{B}\right) b_{a} S \frac{\rho(H) V^{2}}{2}, M(H) \underline{\underline{\text { def }}} \frac{V}{a(h)}
\end{aligned}
$$

2. For variables describing motion:

$V$-Flight velocity tangent to trajectory (with respect of air)

$\mathrm{H}$-Height above mean sea level of a drone flight $L$-Drone path in longitudinal direction $\vartheta$-Pitch angle, i.e., angle between longitudinal drone axis and horizontal plane

$\theta$-Tilting of velocity about horizontal plane $a$-Angle of attack, i.e., angle between longitudinal axis of a drone and projection of drone velocity on the symmetry plane of the drone

3. Drones parameters:
Here

$c_{x}$ and $c_{y}$-coefficients of resistance and carrying forces, correspondently

$m_{z}$-Coefficient of moment

$S$-Area of winds

$b_{a}$-Length of wind chord

$\rho(H)$ - Air density at a flight height

$M=V / a-$ Mach number

$a$-Sound velocity

$m_{z}\left(\alpha, \alpha, \dot{\vartheta}, V, \delta_{B}, \rho\right)=m_{1 z}\left(\alpha, M(V, H), \delta_{B}\right)+m_{2 z}(M(V, H), \dot{\alpha}, \dot{\vartheta})$

Aerodynamical damping moment:

$m_{2 z}(M(V, H), \dot{\alpha}, \dot{\vartheta})=k\left(\frac{L_{1}}{M(V, H)} \dot{\vartheta}+k^{\prime} \dot{\alpha}\right)$

$L_{1}$-Distance from tail unit to center of mass $k, k^{\prime}$-Constants 
Dependence of parameters on a flight height above mean sea level is defined by the following equations:

$\rho(H)=\rho(0)\left(\frac{T_{H}(H)}{T(0)}\right)^{\frac{1}{\gamma-1}} ; a(H)=\sqrt{\gamma R T_{H}(H)} ; T_{H}(H)=T(0)-\beta H$,

where

$T_{H}(H)$ - Temperature at a flight height

$T(0), \rho(0)$-Temperature and air density at mean sea level

$\gamma$-Adiabatic constant

$R$-Gas constant

$\beta$-Temperature gradient over height

$G \sin \left(\theta_{0}\right)=P_{0} \cos \left(\alpha_{0}\right)-\left(c_{x}\right)_{0} S \frac{\rho_{0} V_{0}^{2}}{2} \stackrel{\text { def }}{=}-\frac{1}{2}\left(c_{x}^{\prime}\right)_{0} S \rho_{0} V_{0}^{2}$

$G \cos \left(\theta_{0}\right)=P_{0} \sin \left(\alpha_{0}\right)+\left(c_{y}\right)_{0} S \frac{\rho_{0} V_{0}^{2}}{2} \stackrel{\text { def }}{=} \frac{1}{2}\left(c_{y}^{\prime}\right)_{0} S \rho_{0} V_{0}^{2}$

$\left(m_{z}\right)_{0}=0$

$V_{0} \sin \left(\theta_{0}\right)=-\left(U_{y}\right)_{0}$

$L(t)=\left(V \cos \left(\theta_{0}\right)+\left(U_{x}\right)_{0}\right) t+L(0)$

$\theta_{0}=\vartheta_{0}-\alpha_{0}$

$\delta_{p}(t)=0 ; \delta_{B}(t)=0 ; f_{1}(t)=0 ; f_{2}(t)=0 ; f_{3}(t)=0 ; \Delta U_{x}(t)=0 ; \Delta U_{y}(t)=0$

\subsubsection{Stationary desirable trajectory}

2.2.2.1 Solution of nonlinear equation For the constant wind, zero-controlling external small random forces and moments, we can find a steady-state solution for a drone flight:

$V_{0}, \theta_{0}, \alpha_{0}, \vartheta_{0}, H_{0},\left(U_{x}\right)_{0},\left(U_{y}\right)_{0}$

$U_{y}(t)=\left(U_{y}\right)_{0}+\Delta U_{y}(t)$

$U_{x}(t)=\left(U_{x}\right)_{0}+\Delta U_{x}(t)$,

where $\Delta U_{x}(t), \Delta U_{y}(t)$-small wind fluctuations.

The steady-state solution can be obtained from (1) by equating all external small random forces and moments, controlling parameters, wind velocity fluctuations, all derivatives to zero:
Table 1 Coefficients of equations for longitudinal motion

\begin{tabular}{|c|c|c|c|c|c|c|}
\hline & \multicolumn{6}{|l|}{ Drone } \\
\hline & \multirow{2}{*}{$\begin{array}{l}\text { Lightweight } \\
H=11 \mathrm{~km} \\
M=0.9 \\
\tau_{a}=3.8 \mathrm{~s}\end{array}$} & \multicolumn{2}{|l|}{ Middle } & \multicolumn{3}{|l|}{ Heavy } \\
\hline & & $H=0$, landing & $\begin{array}{l}H=4 \mathrm{~km} \\
M=0.65 \\
\tau_{a}=2.1 \mathrm{~s}\end{array}$ & $\begin{array}{l}H=8 \mathrm{~km} \\
M=0.8 \\
\tau_{a}=2.5 \mathrm{~s}\end{array}$ & $\begin{array}{l}H=12 \mathrm{~km} \\
M=0.9 \\
\tau_{a}=4 \mathrm{~s}\end{array}$ & $H=0$, landing \\
\hline$n_{11}$ & 0.024 & 0.12 & 0.019 & 0.026 & 0.048 & 0.12 \\
\hline$n_{12}$ & -0.11 & -0.28 & 0.019 & -0.025 & -0.079 & -0.12 \\
\hline$n_{13}$ & 0.2 & 0.4 & 0.3 & 0.1 & 0.17 & 0.3 \\
\hline$n_{14}$ & $-4.3 \cdot 10^{-4}$ & - & $-4.4 \cdot 10^{-4}$ & $-4 \cdot 10^{-4}$ & $-4.2 \cdot 10^{-4}$ & - \\
\hline$n_{21}$ & -0.4 & -0.8 & -0.6 & -0.36 & -0.68 & -0.65 \\
\hline$n_{22}$ & 2.4 & 2.4 & 2.66 & 3 & 2.4 & 2.35 \\
\hline$n_{23}$ & 0 & 0.02 & 0 & 0 & 0 & 0.015 \\
\hline$n_{24}$ & $-1.22 \cdot 10^{-2}$ & - & $-1.28 \cdot 10^{-2}$ & $-1.1 \cdot 10^{-2}$ & $-1.2 \cdot 10^{-2}$ & - \\
\hline$n_{31}$ & 0 & 0 & 0 & 0 & -1.2 & 0 \\
\hline$n_{0}$ & 0.4 & 0.59 & 0.59 & 1.17 & 0.68 & 0.9 \\
\hline$n_{32}$ & 38 & 6.6 & 10.63 & 42 & 36 & 8 \\
\hline$n_{33}$ & 2.45 & 1.67 & 1.69 & 2.5 & 2.42 & 2.35 \\
\hline$n_{34}$ & -0.053 & - & -0.055 & -0.05 & -0.05 & - \\
\hline$n_{\mathrm{B}}$ & 49 & 15.2 & 24.5 & 28 & 46 & 8.4 \\
\hline$n_{p}$ & 0.022 & 0.019 & 0.021 & 0.02 & 0.02 & 0.019 \\
\hline
\end{tabular}


Here we use the following steady-state parameters:

$H_{0}$ corresponding with steady flight get small increments

$\begin{aligned}\left(T_{H}\right)_{0} & =T_{0}-\beta H_{0} ; \rho_{0}=\rho(0)\left(\frac{T_{H}\left(H_{0}\right)}{T_{0}}\right)^{\frac{1}{\gamma-1}} ; a_{0}=\sqrt{\gamma R\left(T_{H}\right)_{0}} ; M_{0}=\frac{v_{0}}{a_{0}} \\ P_{0} & =P\left(0, M_{0}\right) ;\left(c_{x}\right)_{0}=c_{x}\left(a_{0}, M_{0}\right) ;\left(c_{y}\right)_{0}=c_{y}\left(a_{0}, M_{0}\right) ;\left(m_{z}\right)_{0}=m_{z}\left(a_{0}, M_{0}, 0,0,0\right)\end{aligned}$

$\left(\frac{\partial c_{x}^{\prime}}{\partial M}\right)_{0}\left(\frac{\partial c_{y}^{\prime}}{\partial M}\right)_{0},\left(\frac{\partial c_{x}}{\partial \alpha}\right)_{0^{\prime}}\left(\frac{\partial c_{y}}{\partial \alpha}\right)_{0^{\prime}}\left(\frac{\partial P}{\partial \delta_{P}}\right)_{0}\left(c_{y}^{\prime}\right)_{0^{\prime}}\left(c_{x}^{\prime}\right)_{0^{\prime}}\left(\frac{\partial m_{z}}{\partial \dot{\alpha}}\right)_{0^{\prime}}$ $\left(\frac{\partial m_{z}}{\partial \alpha}\right)_{0}{ }^{\prime}\left(\frac{\partial m_{z}}{\partial \dot{\vartheta}}\right)_{0}{ }^{\prime}\left(\frac{\partial m_{z}}{\partial M}\right)_{0},\left(\frac{\partial m_{z}}{\partial \delta_{B}}\right)_{0}$-values of the functions and

$\Delta \vartheta, \Delta \theta, \Delta V, \Delta \alpha, \Delta H$ caused by perturbations action on a flight. Let us define the following deviations from the stationary path:

its derivatives for the steady values $V_{0}, \theta_{0}, \alpha_{0}, \vartheta_{0}, H_{0},\left(U_{x}\right)_{0^{\prime}}\left(U_{y}\right)_{0^{\prime}} \delta_{B}(t)=0, \delta_{P}(t)=0$

$V=V_{0}+\Delta V(t)$

$\theta=\theta_{0}+\Delta \theta$

$\vartheta=\vartheta_{0}+\Delta \vartheta(t)$

2.2.2.2 Choosing desirable parameters for stationary solu-

$\alpha=\alpha_{0}+\Delta \alpha(t)$ tion The typical real values of the coefficients $n_{i j}$ are given $H=H_{0}+\Delta H(t)$ in Table 1.

$v(t)=\frac{\Delta V}{V_{0}} ; h(t)=\frac{\Delta H}{V_{0} \tau_{a}} ; \alpha(t)=\Delta \alpha ; \theta(t)=\Delta \theta ; \vartheta(t)=\Delta \vartheta ; v_{y}(t)=\frac{\Delta U_{y}}{V_{0}} ; v_{x}(t)=\frac{\Delta U_{x}}{V_{0}} ;$

\subsubsection{Linear equations for perturbations with respect}

The correspondent parameters are the following: to stationary solution

$$
\tau_{a}=\frac{m}{\rho_{0} V_{0} S} ; \mu=\frac{b_{a} m}{2 r_{z}^{2} \rho_{0} S} ; r_{z}^{2}=\frac{J_{z}}{m}
$$

2.2.3.1 Concluding linear equation for the perturbations Since system (1) is nonlinear, it is too hard to use those equations to analyze stability. We need to linearize those equations on the premise that the parameters $\vartheta_{0}, \theta_{0}, V_{0}, \alpha_{0}$, $r_{z}$-inertial radius

$\bar{t}=\frac{t}{\tau_{a}} ; p=\frac{\mathrm{d}}{\mathrm{d} \bar{t}}$

$c_{x}^{\prime}(M) \underline{\underline{\operatorname{def}}} c_{x}\left(\alpha_{0}, M\right)-2 \frac{P(0, M) \cos \left(\alpha_{0}\right)}{\rho_{0} S V_{0}^{2}} ; c_{y}^{\prime}(M) \underline{\underline{\operatorname{def}}} c_{y}\left(\alpha_{0}, M\right)+2 \frac{P(0, M) \cos \left(\alpha_{0}\right)}{\rho_{0} S V_{0}^{2}} ;$

We can make linearization of (1) in the neighborhood of the found steady-state solution:

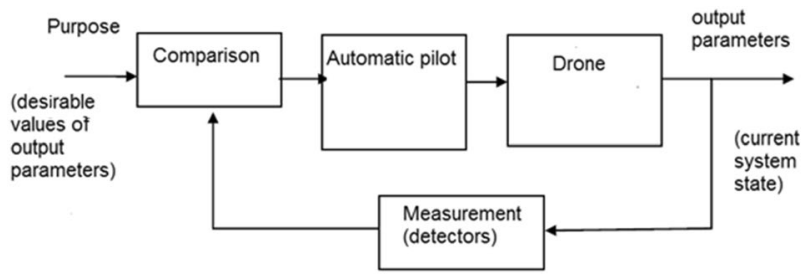

Fig. 2 Automatic control 


$$
\begin{aligned}
& \left(p+n_{11}\right) v+n_{12} \alpha+n_{13} \vartheta+n_{14} h=n_{p} \delta_{p}+f_{1}(t) \\
& -n_{21} v+\left(p+n_{22}\right) \alpha-\left(p+n_{23}\right) \vartheta+n_{24} h=f_{2}(t) \\
& n_{31} v+\left(n_{0} p+n_{32}\right) \alpha+\left(p^{2}+n_{33} p\right) \vartheta+n_{34} h=-n_{B} \delta_{B}(t)+f_{3}(t) \\
& -n_{41} v+n_{42} \alpha-n_{42} \vartheta+p h=v_{y}(t) \\
& n_{11}=\frac{M_{0}}{2}\left(\frac{\partial c_{x}^{\prime}}{\partial M}\right)_{0}+\left(c_{x}\right)_{0} ; \quad n_{12}=\frac{1}{2}\left(\left(\frac{\partial c_{x}}{\partial \alpha}\right)_{0}-\left(c_{y}\right)_{0}\right) ; \\
& n_{13}=\frac{1}{2}\left(c_{y}^{\prime}\right)_{0} ; \quad n_{14}=\frac{\beta V_{0} \tau_{0}}{2\left(T_{H}\right)_{0}}\left[\frac{M_{0}}{2}\left(\frac{\partial c_{x}^{\prime}}{\partial M}\right)^{\prime}-\frac{\left(c_{x}\right)_{0}}{\gamma-1}\right]^{\prime} \\
& n_{21}=-\left(\frac{M_{0}}{2}\left(\frac{\partial c_{y}^{\prime}}{\partial M}\right)+\left(c_{y}\right)_{0}\right) ; \quad n_{22}=\frac{1}{2}\left(\left(\frac{\partial c_{y}}{\partial \alpha}\right)_{0}+\left(c_{x}\right)_{0}\right) ; \\
& n_{23}=\frac{1}{2}\left(c_{x}^{\prime}\right)_{0} ; \quad n_{24}=\frac{\beta V_{0} \tau_{0}}{2\left(T_{H}\right)_{0}}\left[\frac{M_{0}}{2}\left(\frac{\partial c_{y}^{\prime}}{\partial M}\right)_{0}-\frac{\left(c_{y}\right)_{0}}{\gamma-1}\right] ; \\
& n_{31}=-\mu M_{0}\left(\frac{\partial m_{z}}{\partial M}\right)_{0} ; \quad n_{32}=-\mu\left(\frac{\partial m_{z}}{\partial \alpha}\right)_{0} ; \\
& n_{33}=-\frac{\mu}{\tau_{\alpha}}\left(\frac{\partial m_{z}}{\partial \dot{\vartheta}}\right)_{0} ; \quad n_{34}=-\mu \frac{\beta \tau_{\alpha} V_{0}}{2\left(T_{H}\right)_{0}} M_{0}\left(\frac{\partial m_{z}}{\partial M}\right)_{0} \\
& n_{0}=-\frac{\mu}{\tau_{\alpha}}\left(\frac{\partial m_{z}}{\partial \dot{\alpha}}\right)_{0}^{;} \\
& n_{41}=\sin \left(\theta_{0}\right) ; \quad n_{42}=\cos \left(\theta_{0}\right) ; \\
& n_{p}=\frac{\left(\frac{\partial P}{\partial \delta_{p}}\right)_{0} \cos \left(\alpha_{0}\right)}{\rho_{0} s V_{0}^{2}} ; \quad n_{B}=-\mu\left(\frac{\partial m_{z}}{\partial \delta_{B}}\right)_{0} ; \quad p=\frac{d}{d \bar{t}}
\end{aligned}
$$

2.2.3.2 Description of controlled parameters in linear equations defined by autopilot For the case when stationary parameters cannot provide stability of the desirable stationary trajectory themselves, we need to use autopilots (Fig. 2). An autopilot states the controlling parameters $\delta_{p}, \delta_{B}$ to be functions of the output-controlled parameters $(v(t) ; h(t) ; \alpha(t) ; \vartheta(t))$ :

$$
\begin{aligned}
& \delta_{P}(t-\tau)=p_{1} v(t-\tau)+p_{2} \alpha(t-\tau)+p_{3} \vartheta(t-\tau)+p_{4} h(t-\tau) \\
& \delta_{B}(t-\tau)=b_{1} v(t-\tau)+b_{2} \alpha(t-\tau)+b_{3} \vartheta(t-\tau)+b_{4} h(t-\tau)
\end{aligned}
$$

which are deviations from the desirable stationary trajectory. The values of the output parameters can be obtained by autopilot from navigation measurements, for example, from vision-based navigation, inertial navigation, satellite navigation, and so on. On the basis of these navigation measurements, the autopilot forms controlling signals to decrease undesirable deviation. Unfortunately, there always exists some time delay $\tau$ in getting information about the output-controlled parameters to autopilot for any navigation measurements. So, we have a problem, because of the lack of some necessary information for controlling. In this paper, we demonstrate that we are possible even for such conditions with the time delay to get controlling signal providing a stable flight.

\section{Analysis of drone flight stability}

\subsection{Adjusting the system of linear differential equation for perturbations to the form appropriate for using preliminary mathematical theory}

We have to adjust our system to a proper form to apply the theory of stability.

$$
\begin{aligned}
v^{\prime}(t)= & -n_{11} v(t)-n_{12} \alpha(t)-n_{13} \vartheta(t) \\
& -n_{14} h(t)=n_{p} \delta_{p}(t-\tau) \\
\alpha^{\prime}(t)= & \vartheta^{\prime}(t)+n_{21} v(t)-n_{22} \alpha(t)+n_{23} \vartheta(t)-n_{24} h(t) \\
\vartheta^{\prime \prime}(t)= & -n_{0} \alpha^{\prime}(t)-n_{33} \vartheta^{\prime}(t)-n_{31} v(t) \\
& -n_{32} \alpha(t)-n_{34} h(t)-n_{B} \delta_{B}(t-\tau) \\
h^{\prime}(t)= & n_{41} v(t)-n_{42} \alpha(t)+n_{42} \vartheta(t)
\end{aligned}
$$

Let us start with linear substitution. It brings the system to a form where all diagonal coefficients are nonzero.

The first substitution is following:

$h(t)=\lambda(t)-W v(t) \Rightarrow h^{\prime}(t)=\frac{d \lambda(t)}{d t}-W \frac{d v(t)}{d t} ;$

Now we have:

$$
\begin{aligned}
v^{\prime}(t)= & -n_{11} v(t)-n_{12} \alpha(t)-n_{13} \vartheta(t)-n_{14}(\lambda(t)-W v(t))=n_{p} \delta_{p}(t-\tau) \\
\alpha^{\prime}(t)= & \vartheta^{\prime}(t)+n_{21} v(t)-n_{22} \alpha(t)+n_{23} \vartheta(t)-n_{24}(\lambda(t)-W v(t)) \\
\vartheta^{\prime \prime}(t)= & -n_{0} \alpha^{\prime}(t)-n_{33} \vartheta^{\prime}(t)-n_{31} v(t)-n_{32} \alpha(t)-n_{34}(\lambda(t)-W v(t))-n_{B} \delta_{B}(t-\tau) \\
\lambda^{\prime}(t)= & W\left(-n_{11} v(t)-n_{12} \alpha(t)-n_{13} \vartheta(t)-n_{14}(\lambda(t)-W v(t))+n_{p} \delta_{p}(t-\tau)\right) \\
& +n_{41} v(t)-n_{42} \alpha(t)+n_{42} \vartheta(t)
\end{aligned}
$$


The second linear substitution is used here to decrease the order of the system.

$\vartheta^{\prime}(t)=\varphi(t)+b_{0} \vartheta(t)$

$$
\begin{aligned}
v^{\prime}(t)= & -n_{11} v(t)-n_{12} \alpha(t)-n_{13} \vartheta(t) \\
& -n_{14}(\lambda(t)-W v(t))+n_{p} \delta_{p}(t-\tau) \\
\alpha^{\prime}(t)= & \varphi(t)+b_{0} \vartheta(t)+n_{21} v(t)-n_{22} \alpha(t) \\
& +n_{23} \vartheta(t)-n_{24}(\lambda(t)-W v(t)) \\
\vartheta^{\prime}(t)= & \varphi(t)+b_{0} \vartheta(t) \\
\varphi^{\prime}(t)= & -n_{B} \delta_{B}(t-\tau)+\left(\left(-n_{23}-b_{0}\right) n_{0}-b_{0}\left(n_{33}+b_{0}\right)\right) \vartheta(t) \\
& +\left(\left(-W n_{24}-n_{21}\right) n_{0}+W n_{34}-n_{31}\right) v(t) \\
& +\left(-n_{33}-b_{0}-n_{0}\right) \varphi(t)+\left(n_{0} n_{24}-n_{34}\right) \\
& \lambda(t)-\alpha(t)\left(-n_{0} n_{22}+n_{32}\right) \\
\lambda^{\prime}(t)= & W \delta_{p}(t-\tau) n_{p}+\left(W^{2} n_{14}-W n_{11}+n_{41}\right) \\
& v(t)+\left(-W n_{13}+n_{42}\right) \vartheta(t) \\
& +\left(-W n_{12}-n_{42}\right) \alpha(t)-W \lambda(t) n_{14}
\end{aligned}
$$

$\delta_{P}$ and $\delta_{B}$ are control parameters. We want to express them as linear combinations of our original variables:

$\delta_{P}(t-\tau)=p_{1} v(t-\tau)+p_{2} \alpha(t-\tau)+p_{3} \vartheta(t-\tau)+p_{4} h(t-\tau) ;$ $\delta_{B}(t-\tau)=b_{1} v(t-\tau)+b_{2} \alpha(t-\tau)+b_{3} \vartheta(t-\tau)+b_{4} h(t-\tau)$

Taking this into account the system will be the following:

$$
\begin{aligned}
v^{\prime}(t)= & -n_{11} v(t)-n_{12} \alpha(t)-n_{13} \vartheta(t)-n_{14}(\lambda(t)-W v(t)) \\
& +n_{p}\left(p_{1} v(t-\tau)+p_{2} \alpha(t-\tau)+p_{3} \vartheta(t-\tau)+p_{4}((\lambda(t-\tau)-W v(t-\tau)))\right. \\
\alpha^{\prime}(t)= & \varphi(t)+b_{0} \vartheta(t)+n_{21} v(t)-n_{22} \alpha(t)+n_{23} \vartheta(t)-n_{24}(\lambda(t)-W v(t)) \\
\vartheta^{\prime}(t)= & \varphi(t)+b_{0} \vartheta(t) \\
\varphi^{\prime}(t)= & -b_{0}\left(\varphi(t)+b_{0} \vartheta(t)\right) \\
& -n_{0}\left(\varphi(t)+b_{0} \vartheta(t)+n_{21} v(t)-n_{22} \alpha(t)+n_{23} \vartheta(t)-n_{24}(\lambda(t)-W v(t))\right) \\
& -n_{33}\left(\varphi(t)+b_{0} \vartheta(t)\right)-n_{31} v(t)-n_{32} \alpha(t)-n_{34}(\lambda(t)-W v(t))-n_{B}\left(b_{1} v(t-\tau)\right) \\
& +b_{2} \alpha(t-\tau)+b_{3} \vartheta(t-\tau)+b_{4}(\lambda(t-\tau)-W v(t)) \\
\lambda^{\prime}(t)= & W\left(p_{1} v(t-\tau)+p_{2} \alpha(t-\tau)+p_{3} \vartheta(t-\tau)+p_{4}(\lambda(t-\tau)-W v(t-\tau))\right) n_{p} \\
& +\left(W^{2} n_{14}-W n_{11}+n_{41}\right) v(t)+\left(-W n_{13}+n_{42}\right) \vartheta(t) \\
& +\left(-W n_{12}-n_{42}\right) \alpha(t)-W \lambda(t) n_{14}
\end{aligned}
$$

Taking other coefficients from the first column of Table 1, we get following:

\subsection{Applying the mathematical theory for stabilization of drone flight with respect to the chosen desirable stationary trajectory}

Apply the condition (1.1) of Theorem 3.2 in [1], described in Sect. 2 of this paper to system (3.1)

Consider the system of inequalities:

$$
\begin{aligned}
& n_{11}=0.024, n_{12}=-0.11, n_{13}=0.2, \\
& n_{14}=-0.00043, n_{21}=-0.4, n_{22}=2.4, \\
& n_{23}=0, n_{24}=-0.0122, n_{31}=0, \\
& n_{32}=38, n_{33}=2.45, \\
& n_{34}=-0.053, n_{0}=0.4, n_{B}=49, \\
& n_{P}=0.022, n_{41}=0, n_{42}=1 ;
\end{aligned}
$$




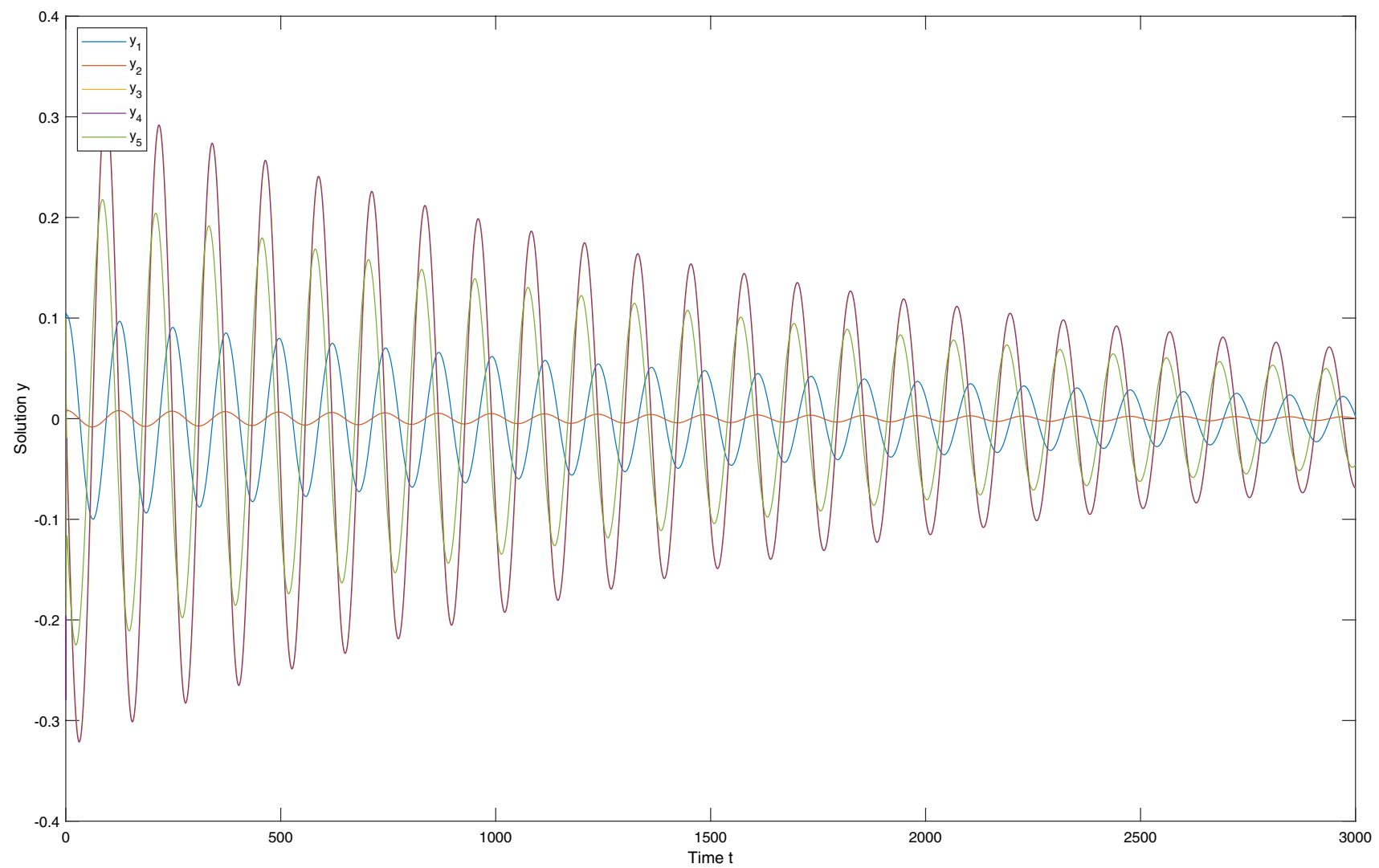

Fig. 3 Numerical simulation of system (3.1) for time delay $\tau=0.447$ (unitless time), where $y_{1}(t)=v(t), y_{2}(t)=a(t), y_{3}(t)=\vartheta(t), y_{4}(t)=\varphi(t)$, $y_{5}(t)=\lambda(t)$

We choose $z_{1}=1 \cdot|z|, z_{2}=1 \cdot|z|, \quad z_{3}=10^{-10} \cdot|z|$, $z_{4}=10^{-10} \cdot|z|, z_{5}=1 \cdot|z|$, where $|z| \gg 1,|z| \cdot 10^{-10} \gg 1$

From (3.5)

$b_{1}=-\frac{n_{21}\left(n_{0}+1\right)}{n_{B}}$

$b_{2}=\frac{n_{0} n_{22}-n_{32}}{n_{B}}$

$b_{3}=-\frac{b_{0}^{2}+n_{0} b_{0}+n_{33} b_{0}+n_{0} n_{32}}{n_{B}}$

$b_{4}=\frac{n_{0} n_{24}-n_{34}}{n_{B}}$

$b_{0} \geq-n_{0}-n_{33}+1 /\left(|z| \cdot 10^{-10}\right)$

From (3.4)

$b_{0} \geq-\left(1+1 /\left(|z| \cdot 10^{-10}\right)\right)$

We choose $b_{0}=-1.001$

Then we can calculate parameters $b_{1}, b_{2}, b_{3}, b_{4}$ :

$b_{1}=0.01142857143, b_{2}=-0.7559183673$,

$b_{3}=0.03777242857, b_{4}=0.0009820408163$
From (3.3)

$$
\begin{gathered}
n_{22}-\left|n_{24} W+n_{21}\right|-\left(\left|n_{23}+b_{0}\right|+1\right) \cdot 10^{-10}-\left|n_{24}\right| \geq \frac{1}{|z|} \\
\frac{n_{22}}{\left|n_{24}\right|}-\frac{\left(\left|n_{23}+b_{0}\right|+1\right)}{\left|n_{24}\right|} \cdot 10^{-10}-1-\frac{1}{|z|\left|n_{24}\right|}-\frac{n_{21}}{n_{24}} \geq W \\
\geq-\frac{n_{22}}{\left|n_{24}\right|}+\frac{\left(\left|n_{23}+b_{0}\right|+1\right)}{\left|n_{24}\right|} \cdot 10^{-10}+1+\frac{1}{|z|\left|n_{24}\right|}-\frac{n_{21}}{n_{24}} \\
162.95-\frac{\left(\left|n_{23}+b_{0}\right|+1\right)}{\left|n_{24}\right|} \cdot 10^{-10}-\frac{1}{|z|\left|n_{24}\right|} \geq W \\
\geq-228.51+\frac{\left(\left|n_{23}+b_{0}\right|+1\right)}{\left|n_{24}\right|} \cdot 10^{-10}+\frac{1}{|z|\left|n_{24}\right|}
\end{gathered}
$$

We choose $W=-63$

We choose

$$
\begin{aligned}
& \left|-n_{p} p_{2}+n_{12}+\frac{n_{42}}{W}\right|=\left|-n_{p} p_{2}+n_{12}\right|=\left|\frac{n_{42}}{2 W}\right| \\
& -n_{p} p_{3}+n_{13}-\frac{n_{42}}{W}|=|-n_{p} p_{3}+n_{13}|=| \frac{n_{42}}{2 W} \mid
\end{aligned}
$$




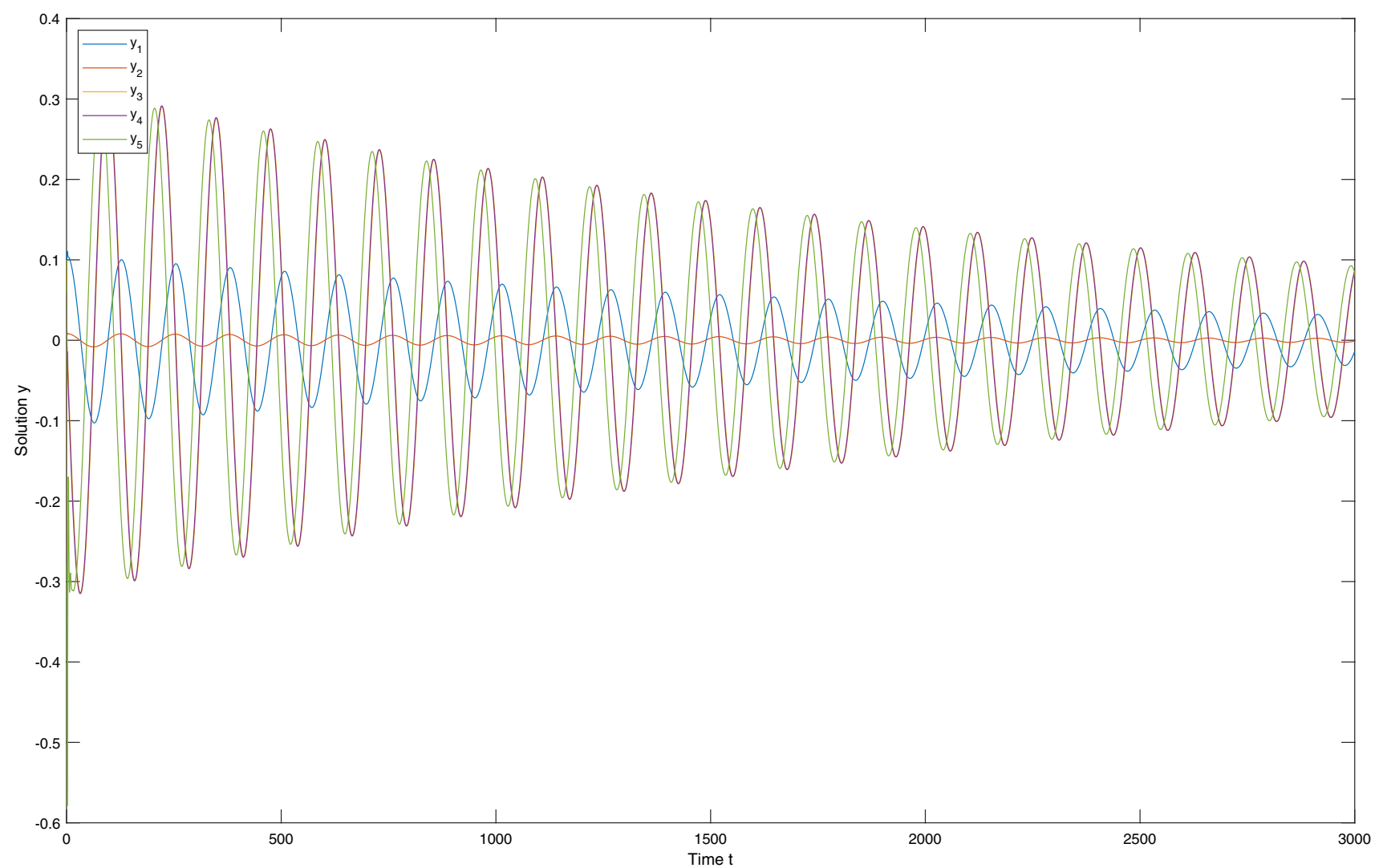

Fig. 4 Numerical simulation of system (3.1) for time delay $\tau=1$ (unitless time), where $y_{1}(t)=v(t), y_{2}(t)=a(t), y_{3}(t)=\vartheta(t), y_{4}(t)=\varphi(t), y_{5}(t)=\lambda(t)$

So

$p_{2}=-\frac{\left|\frac{n_{42}}{2 W}\right|+\left|n_{12}\right|}{n_{p}}, p_{3}=-\frac{\left|\frac{n_{42}}{2 W}\right|+\left|n_{13}\right|}{n_{p}} ;$

Then we can calculate parameters $p_{2}, p_{3}$ :

$p_{2}=-5.360750359, p_{3}=9.451659450$

We introduce new variable $p_{5}=p_{1}-W p_{4}$. Substituting $p_{1}=p_{5}+W p_{4}$, we get conditions for parameters $p_{4}$ and $p_{5}$ from (3.2) and (3.6): $p_{1}=-35, p_{4}=0.5512345678$

Now it is possible to find the requirements for delay: We apply the conditions (1.2) of Theorem 3.2 in [1], described in Sect. 2 of this paper to system (3.1).

From equation for $v^{\prime}(t)$, we get two conditions.

The first condition is $\left(n_{14} W+n_{11}-n_{p} p_{1}\right) \tau \leq \frac{1}{e}$. From this condition, we get that $0.821 \tau \leq 0.368$ and finally we have $\tau \leq 0.448$.

$$
\begin{aligned}
& \left(n_{14} W-\left|\frac{n_{42}}{2 W}\right|\left(1+10^{-10}\right)+n_{11}\right)-n_{p} p_{5}-\left|-n_{p} p_{4}+n_{14}\right|>0 \\
& \left(n_{14} W-\left|\frac{n_{42}}{2 W}\right|\left(1+10^{-10}\right)\right)-n_{p} p_{4} W-\left|W^{2} n_{14}+W n_{p} p_{5}-W n_{11}+n_{41}\right|>0
\end{aligned}
$$

$-0.022 p_{5}+0.04315349210-\left|0.022 p_{4}+0.00043\right|>0$ $0.022 p_{4}-0.007506507971-\left|0.00309+0.022 p_{5}\right|>0$

We find the parameters and choose the particular ones that satisfy the requirements:

\section{SN Applied Sciences}

The second condition $\left(n_{14} W+n_{11}-n_{p} p_{1}-\left|n_{p} p_{4} W\right|\right)$ $\tau \leq \frac{1}{e}$. From this condition, we get that $0.057 \tau \leq 0.368$ and finally, $\tau \leq 6.445$.

From equation for $\lambda^{\prime}(t)$, we get that $\left(n_{14} W-n_{p} p_{4} W\right) \tau \leq \frac{1}{e}$. It means that $0.791 \tau \leq 0.368$ and finally, we get $\tau \leq 0.465$. 


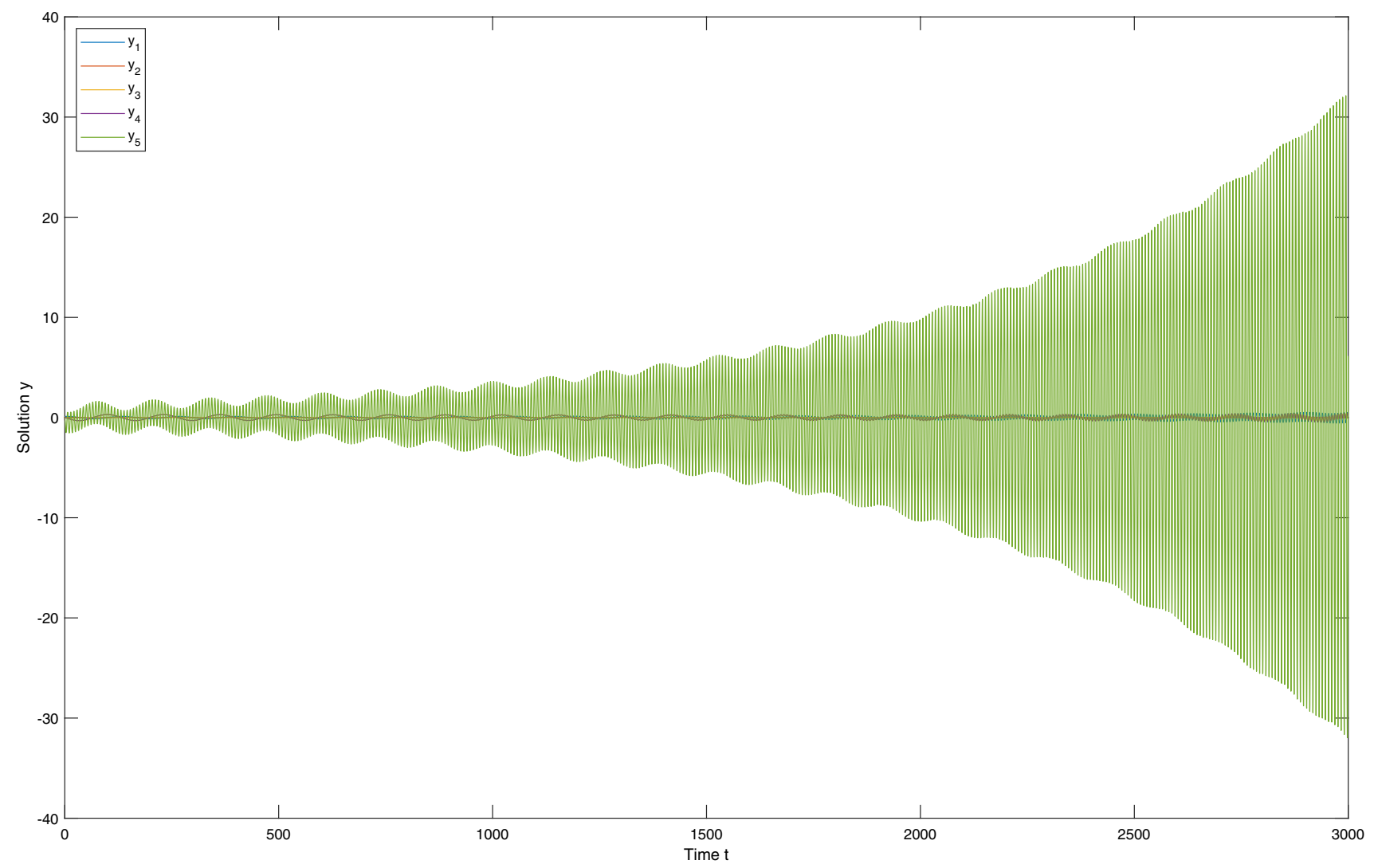

Fig. 5 Numerical simulation of system (3.1) for time delay $\tau=2$ (unitless time), where $y_{1}(t)=v(t), y_{2}(t)=a(t), y_{3}(t)=\vartheta(t), y_{4}(t)=\varphi(t), y_{5}(t)=\lambda(t)$

Finally, from three conditions above, we get the following condition:

$\tau \leq 0.448$.

It is unitless time normalized by $\tau_{a}=3.8 \mathrm{~s}$. Delay time in seconds is defined by

$\tau_{s} \leq 0.448 \tau_{a}=0.448 * 3.8 \mathrm{~s}=1.703 \mathrm{~s}$.

\subsection{Comparing theoretical results with computer simulations}

We made numerical simulation of system (3.1) for different values of time delay (Figs. 3, 4 and 5) and for the controlled parameters of autopilot, which are chosen in the chapter above.

From the mathematical theory, we know that such a system must be stable for time delay satisfying condition (3.8). Indeed, we see from Fig. 3 that initial perturbations of stationary solution decrease in time as it was predicted by theory.

If the system has the same controlled parameters of autopilot, but time delay larger then upper boundary in
(3.8), the mathematical theory cannot give any prediction. Indeed, condition (3.8) is sufficient, but not necessary. Numerical simulations demonstrate that system is stable even for larger values of time delay (see Fig. 4). However, for large enough values of time delay, the system begins to diverse (see Fig. 5). This result demonstrates that our upper boundary for time delay is correct but can be improved.

\section{Conclusion}

We demonstrated possibility to supply stable drone automatic flight when there exists a time delay in transfer of information (about motion parameters) from navigation measurement devices to autopilot. We found controlled parameters for some cases of flight and estimated upper boundary of time delay for such system. Numerical simulations demonstrate that our estimation of upper boundary for time delay is correct but can be improved. It is an open problem for future investigation. 


\section{Compliance with ethical standards}

Conflict of interest On behalf of all authors, the corresponding author states that there is no conflict of interest.

\section{References}

1. Domoshnitsky A, Fridman E (2016) A positivity-based approach to delay-dependent stability of systems with large time-varying delays. Syst Control Lett 97:139-148

2. Agarwal RP, Berezansky L, Braverman E, Domoshnitsky A (2011) Nonoscillation theory of functional differential equations with applications. Springer, Berlin

3. Agarwal RP, Domoshnitsky A, Maghakyan A (2015) On exponential stability of second order delay differential equations. Czechoslov Math J 65(140):1047-1068. https://doi.org/10.1007/ s10587-015-0227-9

4. Domoshnitsky A (2014) Nonoscillation, maximum principles, and exponential stability of second order delay differential equations without damping term. J Inequal Appl 2014:361. https://doi.org/10.1186/1029-242X-2014-361

5. Domoshnitsky A, Maghakyan A, Berezansky L (2017) W-transform for exponential stability of second order delay differential equations without damping terms. J Inequal Appl 2017:20

6. Domoshnitsky A (2001) Unboundedness of solutions and instability of second order equations with delayed argument. Differ Integral Equ 14:559-576

7. Samoilov LK (2016) Classical method of the account of influence time delays of signals in devices of control systems. Izvestiya SFedU Eng Sci 4(177):40-49

8. Krushel'EG, Stepanchenko IV (2004) Informacionnoe zapazdyvanie $v$ cifrovyh sistemah upravlenija. Monograph, VolgGTU, Volgograd, p 124

9. Araki M (2009) PID control. In: Unbehauen H (ed) Control systems, robotics and automation, vol II. Eolss Publishers/UNESCO, p 58

10. Azbelev NV, Simonov PM (2003) Stability of differential equations with aftereffect. Stability and control: theory, methods and applications, vol 20. CRC Press, London

11. Kupervasser $O$, Rubinstein A (2013) Correction of inertial navigation on system's errors by the help of video-based navigator based on digital terrain map. Positioning 4:89-108
12. Kupervasser O, Lerner R, Rivlin E, Rotstein $\mathrm{H}$ (2008) Error analysis for a navigation algorithm based on optical-flow and a digital terrain map. In: The proceedings of the 2008 IEEE/ION position, location and navigation symposium, pp 1203-1212

13. Kupervasser $\mathrm{O}$, Voronov $\mathrm{V}$ (2011) A navigation filter for fusing DTM/correspondence updates. In: Proceedings of the IEEE international conference on robotics and biomimetics (ROBIO), pp 1591-1596

14. Lerner R, Kupervasser O, Rivlin E (2006) Pose and motion from omnidirectional optical flow and a digital terrain map. In: The proceedings of the 2006 IEEE/RSJ international conference on intelligent robots and systems, pp 2251-2256

15. Kupervasser OYu, Voronov VV (2012) Correction of inertial navigation system's errors by the help of video-based navigator based on digital terrarium map. In: Presented at XIX St.-Petersburg international conference on the integrated navigational systems (MKINS2012)

16. Lerner R, Rivlin E, Rotstein HP (2006) Pose and motion recovery from correspondence and a digital terrain map. IEEE Trans Pattern Anal Mach Intell 28(9):1404-1417

17. Kupervasser O, Sarychev V, Rubinstein A, Yavich R (2018) Robust positioning of drones for land use monitoring in strong terrain relief using vision-based navigation. Int J Geomate 14(45):10-15

18. Liu Y, Rodrigues MA (2001) Statistical image analysis for pose estimation without point correspondences. Pattern Recogn Lett 22:1191-1206

19. David P, DeMenthon D, Duraiswami R, Samet H (2002) SoftPOSIT: simultaneous pose and correspondence determination. ECCV 2002, LNCS 2352, pp 698-714. https://doi.org/10.1007/3-54047977-5 46

20. Shin D, Park SG, Song BS, Kim ES, Kupervasser O, Pivovartchuk D, Gartseev I, Antipov O, Kruchenkov E, Milovanov A, Kochetov A, Sazonov I, Nogtev I, Hyun SW (2014) Precision improvement of MEMS gyros for indoor mobile robots with horizontal motion inspired by methods of TRIZ. In: Proceedings of 9th IEEE international conference on nano/micro engineered and molecular systems (IEEE-NEMS 2014) April 13-16, 2014, Hawaii, USA, pp 102-107

21. Bodner VA, Kozlov MS (1961) Stabilization of aerial vehicles and autopilots. Oborongiz, Mscow (in Russian)

Publisher's Note Springer Nature remains neutral with regard to jurisdictional claims in published maps and institutional affiliations. 